\title{
Single-shot measurement of phase and topological properties of orbital angular momentum radiation through asymmetric lateral coherence
}

\author{
B. Paroli, ${ }^{*}$ M. Siano, L. Teruzzi, and M. A. C. Potenza \\ Dipartimento di Fisica, Universitá degli Studi di Milano, via G. Celoria, 16, 20133 Milano, Italy
}

(Received 30 November 2018; published 27 March 2019)

\begin{abstract}
We show a single-shot technique to measure topological and phase properties of radiation carrying orbital angular momentum. The single-shot method is effectively described as the one-dimensional case of a more general two-dimensional approach based on scanning interferometry (asymmetric lateral coherence). The validity of the method has been experimentally verified and the applicability to ultrarelativistic sources of hard x-rays has been discussed. The method is suitable to characterize phase and topological properties of x-ray sources by using simple apertures.
\end{abstract}

DOI: 10.1103/PhysRevAccelBeams.22.032901

\section{INTRODUCTION}

Exotic radiation beams named optical vortices originate when light carries an orbital angular momentum (OAM), a quantity which is intrinsically different from, and independent of, the polarization state of light. At a single photon level, the former corresponds to the quantized momentum $L_{z}=l \hbar$, where $l$ is an integer, while the latter is a manifestation of the intrinsic angular momentum due to the photon spin. From a classical point of view OAM radiation exhibits helical wavefronts and is consequently endowed with an azimuthal phase dependence of the associated scalar field $E \propto e^{i l \theta}$ (on every $x y$-plane perpendicular to the propagation direction $z$ ), as formally defined e.g., by the Laguerre-Gauss modes [1]. The quantity $l$ is known as the topological charge of the vortex beam.

The importance of this peculiar property of light comes from the huge impact that it is having on different fields of research such as telecommunication, quantum information and computing, microscopy, as well as physics and astrophysics. Among the most recent remarkable results, a few are worth mentioning: bandwidth information has been increased by encoding $l$ in the transmitted beam [2], resolution has been pushed beyond the diffraction limit [3], nanoparticles can be finely controlled and manipulated [4], high contrast coronagraphy can be effectively implemented in astrophysics research [5], and novel photoelectric effect rules have been evidenced [6].

\footnotetext{
*bruno.paroli@unimi.it

Published by the American Physical Society under the terms of the Creative Commons Attribution 4.0 International license. Further distribution of this work must maintain attribution to the author(s) and the published article's title, journal citation, and DOI.
}

Despite the fact that vortices can be generated in the visible range by means of holographic or refractive techniques by using lasers, the practical realization of high brilliance $\mathrm{x}$-ray vortices is an open problem, the main limitation being related to the damage threshold of the optical components. Recent works have demonstrated the possibility to generate OAM radiation in the hard $\mathrm{x}$-ray region by exploiting interaction between a high power laser carrying orbital angular momentum and a counterpropagating ultrarelativistic electron beam through Thomson or inverse-Compton interactions [7]. Helical undulators naturally generate circularly polarized radiation and orbital angular momentum radiation due to the spiral motion of electrons induced by the helically structured magnetic field. Recent results explicitly show that the harmonic components of an electromagnetic field radiated by electrons in circular or spiral motion naturally have a helical phase structure, which suggests the presence of orbital angular momentum. Such results have been demonstrated experimentally by observing helical undulator radiations [8]. More recently it has been shown that twisted photons in the Mev energy range can be provided by channeling radiation $[9,10]$.

Aside from the generation issues, such high photon energies pose absolutely new challenges for the development of measuring methods. In this view we have recently developed a scanning interferometric technique called asymmetric lateral coherence (ALC) which has been proven to reliably diagnose OAM radiation from stable sources ranging from the visible to the x-rays [11]. We show here a fundamental improvement of the ALC technique, namely the feasibility of a single shot characterization of OAM sources to drastically reduce the measurement times and storage resource usage. A bench test experiment scaled to visible light is described and extension to x-ray wavelengths is also discussed. 


\section{ASYMMETRIC LATERAL COHERENCE TECHNIQUE IN SINGLE-SHOT}

The asymmetric lateral coherence technique has been recently reported to provide one-dimensional [12-16] and 2-dimensional $[17,18]$ mapping of the real part of the complex degree of coherence $\operatorname{Re} \gamma_{c}$ along linear paths. It is based on a scanning interferometry scheme that exploits an array of double-slits with different spacing $\Delta x$ controlled by a 2 -axis linear stage synchronized with a tracking charge couple device (CCD).

As shown in Fig. 1(a) the scan is performed by moving each slit across the transverse section of the vortex along an horizontal path. The positions of the first and second slits $x_{0}=p-\Delta x / 2$ and $x=p+\Delta x / 2$ are defined by using the scan variables $p$ and $\Delta x$, where $p$ is the position of the double-slit center along the horizontal axis.

For each couple of scan variables $\left(x_{0}, x\right)$ the corresponding interferogram is acquired and processed to extract the horizontal profile at the vertical off-axis position $y_{0}$ as in Fig. 1(b). From the values of the different profiles at the horizontal position corresponding to the center of the double-slits one can build the 2-dimensional

(a)

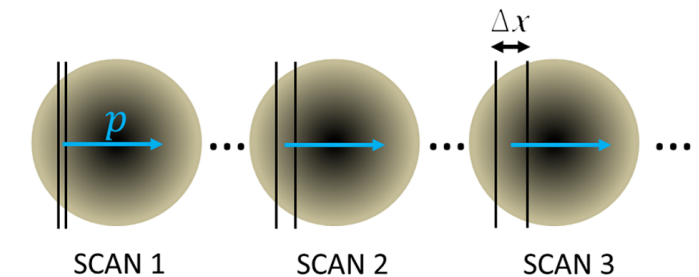

(b)
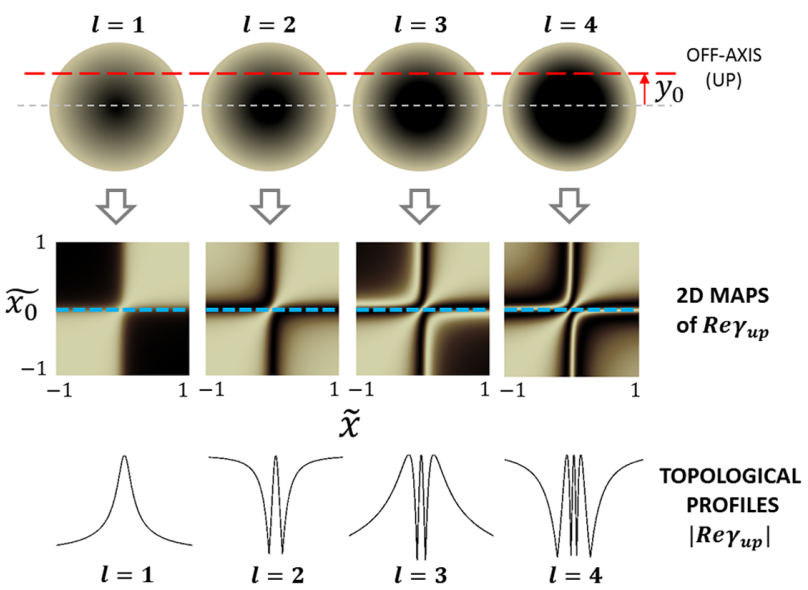

FIG. 1. (a) Sketch of the scan method. The two-dimensional maps are obtained by using a set of double-slits with increasing spacing $\Delta x$. A scan is performed by moving each doubleslit across the cross section of the vortex beam, where $p$ is the double-slit center. (b) Representation of the 2D maps $\left[\operatorname{Re} \gamma_{c}\left(\tilde{x}, \tilde{x_{0}}\right)\right]$ for several topological charges obtained with the ALC technique (white $=1$, black $=-1$ ). The analysis is performed along the off-axis linear path shown by the red dashed line. The topological profiles (bottom) are derived from the maps along the blue dashed line $\tilde{x_{0}}=0$. maps of the asymmetric lateral coherence for $\operatorname{Re} \gamma_{c}$, as shown in the second row of Fig. 1(b).

For a Laguerre-Gauss beam observed in the focal plane, $\operatorname{Re} \gamma_{c}$ can be written as a function of the slit positions $x_{0}, x$ along the linear path at the off-axis position $y_{0}$ [as shown by the red dashed line in Fig. 1(b)] as [11]

$$
\begin{aligned}
\operatorname{Re} \gamma_{c}\left(x, x_{0}\right)= & \operatorname{Re}\left\{\operatorname { e x p } \left[i l \left(\arccos \frac{x}{\sqrt{x^{2}+y_{0}^{2}}}\right.\right.\right. \\
& \left.\left.\left.-\arccos \frac{x_{0}}{\sqrt{x_{0}^{2}+y_{0}^{2}}}\right)\right]\right\} .
\end{aligned}
$$

Examples of the 2-dimensional maps $\operatorname{Re} \gamma_{c}$ derived by Eq. (1) are shown in Fig. 1(b) for topological charges $l=1$, $l=2, l=3$, and $l=4$ as a function of the dimensionless variables $\tilde{x_{0}}=x_{0} / x_{M}$ and $\tilde{x}=x / x_{M}$, where $x_{M}$ is the maximum $x$ value. Although phase and topological properties can be derived from the features of the 2-dimensional maps, a straightforward interpretation can be given to the topological profiles $\left|\operatorname{Re} \gamma_{c}\left(x, x_{0}=0\right)\right|$ of the ALC maps: the number of extrema is $N=2 l-1$, thus yielding a direct measurement of the OAM topological charge, while the phase can be retrieved by using the arccosine function of $\operatorname{Re} \gamma_{c}$ as described in Ref. [11].

Despite the useful information provided by the topological profiles, stable sources are necessary due to the relatively long time scan (several minutes), while a singleshot technique would be preferable for short-time measurements. We show here how to measure in single-shot the topological profiles exploiting a single horizontal doubleslit with finite extension. More precisely, we will show that the topological profiles of the $2 \mathrm{D}$ maps, measured along a linear path at a distance $y_{0}$ from the singularity, are related to the real part of the complex degree of coherence $\operatorname{Re} \gamma_{\text {slit }}$ measured using a double-slit with spacing $\Delta x=2 y_{0}$ oriented along the horizontal direction and centered on the singularity. Explicitly we write (see the Appendix for derivation)

$$
\begin{aligned}
\left|\operatorname{Re} \gamma_{u p}\left(x, x_{0}=0\right)\right| & =\left|\operatorname{Re} \gamma_{d w}^{*}\left(x, x_{0}=0\right)\right| \\
& =\frac{1}{\sqrt{2}} \sqrt{1+\frac{\operatorname{Re} \gamma_{\text {slit }}(x)}{\cos (l \pi)}}
\end{aligned}
$$

where $\operatorname{Re} \gamma_{u p}\left(x, x_{0}=0\right)$ and $\operatorname{Re} \gamma_{d w}^{*}\left(x, x_{0}=0\right)$ are the topological profiles of the ALC maps obtained at the off-axis positions $+y_{0}$ and $-y_{0}$, respectively.

The quantity $\operatorname{Re} \gamma_{\text {slit }}(x)$ is experimentally obtained from the interference pattern $P(x)$ measured along the doubleslit center on the observation plane (see green dashed line in Fig. 2) as

$$
\operatorname{Re} \gamma_{\text {slit }}(x)=\frac{P(x)}{I(x)}-1
$$




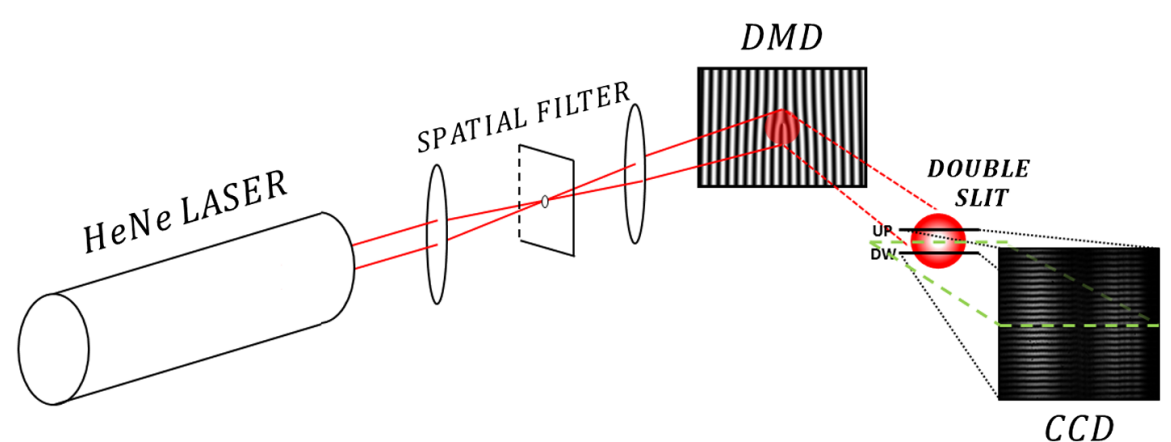

FIG. 2. Experimental setup of the ALC technique in single-shot. A fixed double-slit is centered on the vortex singularity at the detection plane. The first and second slits ( $u p$ and $d w$ ) are at distances $y_{0}$ and $-y_{0}$ from the singularity, respectively. The interference pattern $P(x)$ is acquired at the observation plane (plane of the CCD) along the green dashed line.

Here $I(x)=I_{u p}(x)+I_{d w}(x)$ and $I_{u p}, I_{d w}$ are the intensities from the horizontal slits above and below the singularity, respectively. $I_{u p}(x)=I_{d w}(x)$ assuming the azimuthal symmetry of the vortex. Equation (2) holds for spatially coherent radiation $\left|\gamma_{u p}\right|=\left|\gamma_{d w}\right|=1$ (a condition which is certainly fulfilled for the case of laser light treated in the following section) and has been verified with the experimental setup discussed here below.

\section{EXPERIMENTAL SETUP}

The single-shot method has been proved in the testbench experiment depicted in Fig. 2. Vortices with charge $l=1$ and $l=2$ were generated by digital holograms obtained with a DLP6500 digital micromirror device (DMD). The DMD was illuminated by a spatially filtered, collimated HeNe laser beam $(\lambda=632.8 \mathrm{~nm})$. The optical vortex was sent to a horizontal double-slit centered on the singularity and the corresponding interference pattern was acquired on the observation plane with a cooled low noise $\mathrm{CCD}$ positioned at $45 \mathrm{~cm}$ from the double-slit. The singleshot method was tested with a set of $50 \mu \mathrm{m}$ wide doubleslits with spacing $0.96,1.16,1.20,1.36,1.40,1.56$, and $1.72 \mathrm{~mm}$. The spacing should be chosen to reach a compromise between the instrumental sensitivity and the observable range of the azimuthal angles of the vortex. More precisely, by decreasing the separation the intensity
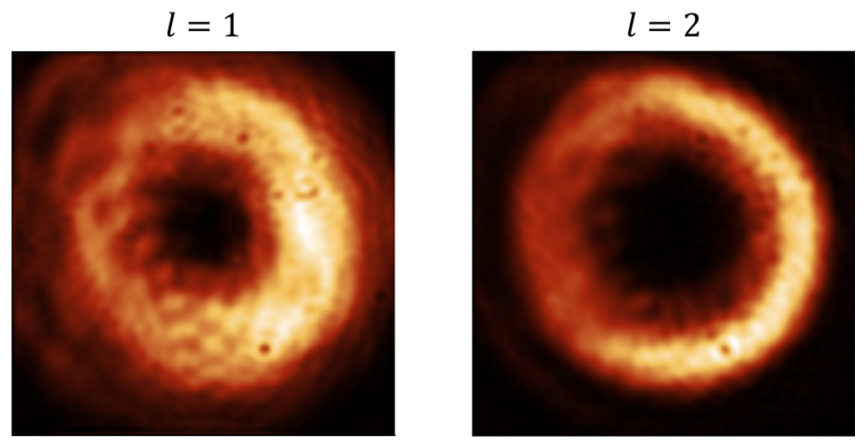

FIG. 3. Intensity profiles of the vortex $l=1$ and $l=2$. decreases due to the hollow-core intensity distribution close to the singularity, conversely the angular width subtended by the slit increases as $\Delta \theta=2 \arctan (L / \Delta X)$, where $L$ is the slit length. The intensity profiles of the vortices shown in Fig. 3 were acquired by sending the beams directly to the CCD after a proper attenuation.

\section{RESULTS AND DISCUSSIONS}

$\operatorname{Re} \gamma_{\text {slit }}$ has been obtained from the interference pattern by using Eq. (3), where $I(x)$ is proportional to the intensity profile $I_{\mathrm{CCD}}$ of the vortex measured by the $\mathrm{CCD}$ along the horizontal path at the off-axis position $y=y_{0}$, $I(x)=A I_{\mathrm{CCD}}(x)$. The unknown constant $A$ has been derived by means of the aforementioned constraint $\left|\operatorname{Re} \gamma_{\text {slit }}(0)\right|=1$, thus yielding $A=P(0) /\left(2 I_{\mathrm{CCD}}(0)\right)$ for even $l$ and $A=P_{M}(0) /\left(2 I_{\mathrm{CCD}}(0)\right)$ for odd $l$, where $P_{M}(0)$ is the nearest maximum of the interference pattern to $x=0$ along the vertical direction. The topological profile $\left|\operatorname{Re} \gamma_{u p}\right|$, shown in Fig. 4, has been derived from Eq. (2) and has been compared to the expected behavior described in Eq. (1). Data are in good agreement with theory close to the singularity, while deviations are evident for both $l=1$ and $l=2$ in the outermost regions. In particular, we observe that deviations depend on the slit separation and thus can be ascribed to the imperfect azimuthal symmetry of the intensity profiles, as evident in Fig. 3, which makes the approximation $I_{u p}=I_{d w}$ of Eq. (3) no longer valid. The left-right asymmetry is ascribed to a small misalignment of the holograms (fork) with respect to the center of the Gaussian beam. Furthermore, the sudden drops for the $l=2$ profiles, as highlighted in the yellow regions of Fig. 4, are related to the saturation of the interference patterns. Though undesired, this saturation effect is necessary to increase the signal-to-noise ratio close to the singularity due to the steeper hollow-core intensity distribution at higher topological charges.

This proof-of-principle experiment performed with a stable HeNe laser shows the validity of the method. Nonpure beams can still carry orbital momentum affecting their interaction with matter. To take into account how 

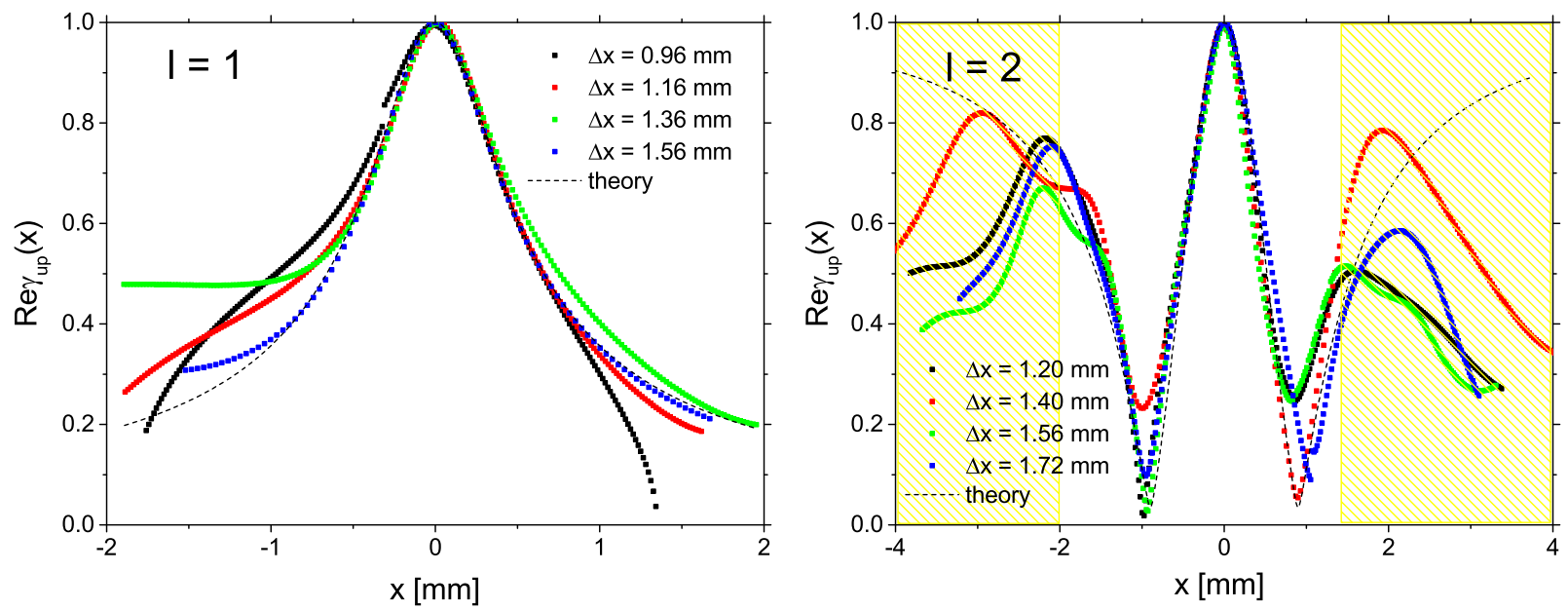

FIG. 4. Single-shot measurements of the topological profiles of the vortex $l=1$ and $l=2$ compared with the theory.

mixtures of beams of different form (e.g., pure Gaussian, Laguerre-Gaussian) affect the analysis, we derive the analogous of Eq. (2) for mixed beams. The uncorrelated superposition of a pure Gaussian beam and a LaguerreGauss beam produces an interference patter $P_{\text {mix }}$ given by:

$$
P_{\text {mix }}(x)=P_{G}(x)+P_{\mathrm{LG}}(x),
$$

where $P_{G}(x)=\left[\operatorname{Re} \gamma_{\text {slit }, G}(x)+1\right] I_{G}(x)$ and $\quad P_{\mathrm{LG}}(x)=$ $\left[\operatorname{Re} \gamma_{\text {slit,LG }}(x)+1\right] I_{\mathrm{LG}}(x)$ are the patterns due to the pure Gaussian and Laguerre-Gauss beams, respectively, and $I_{G}(x), I_{\mathrm{LG}}(x)$ are the intensities measured at the off-axis position $y_{0}$. As in the previous case, here we assume azimuthal symmetry for the intensities. Replacing $P_{G}$ and $P_{\mathrm{LG}}$ in Eq. (4) we find

$$
P_{\text {mix }}(x)=\left(\operatorname{Re}_{\text {mix }}+1\right)\left(I_{G}+I_{\mathrm{LG}}\right)
$$

where

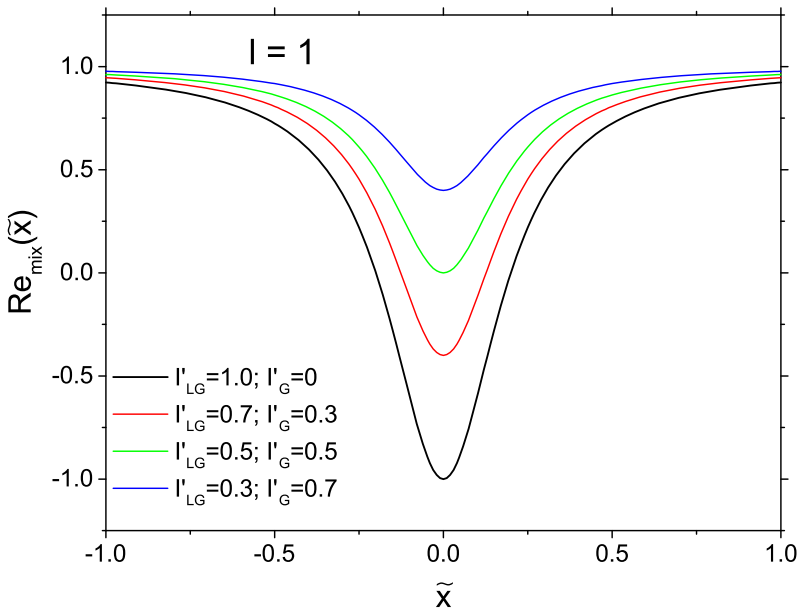

$$
\operatorname{Re}_{\text {mix }}=\frac{\operatorname{Re} \gamma_{\text {slit }, G} I_{G}+\operatorname{Re} \gamma_{\text {slit,LG }} I_{\mathrm{LG}}}{I_{G}+I_{\mathrm{LG}}}
$$

is the weight average of the real part of the complex degrees of coherence of the beams measured along the double-slit center. Of particular interest is the case of coherent beams $\left|\gamma_{\text {slit }, G}\right|=1$ and $\left|\gamma_{\text {slit,LG }}\right|=1$. In fact, replacing the explicit values $\operatorname{Re} \gamma_{\text {slit,G }}$ and $\operatorname{Re} \gamma_{\text {slit,LG }}$ [see Eq. (A12)] in Eq. (6) we find

$\operatorname{Re} \gamma_{u p, \mathrm{LG}}=\frac{1}{\sqrt{2}} \sqrt{\frac{\operatorname{Re}_{\mathrm{mix}}\left(I_{G}+I_{\mathrm{LG}}\right)-\cos (l \pi)\left(I_{G}-I_{\mathrm{LG}}\right)}{I_{\mathrm{LG}} \cos (l \pi)}}$.

Equation (7) shows that the topological profiles of the Laguerre-Gauss beam directly depend on the weight average $\mathrm{Re}_{\text {mix }}$ and on the intensities $I_{G}$ and $I_{\mathrm{LG}}$. In Fig. 5 we show the $\mathrm{Re}_{\text {mix }}$ for several values of the relative

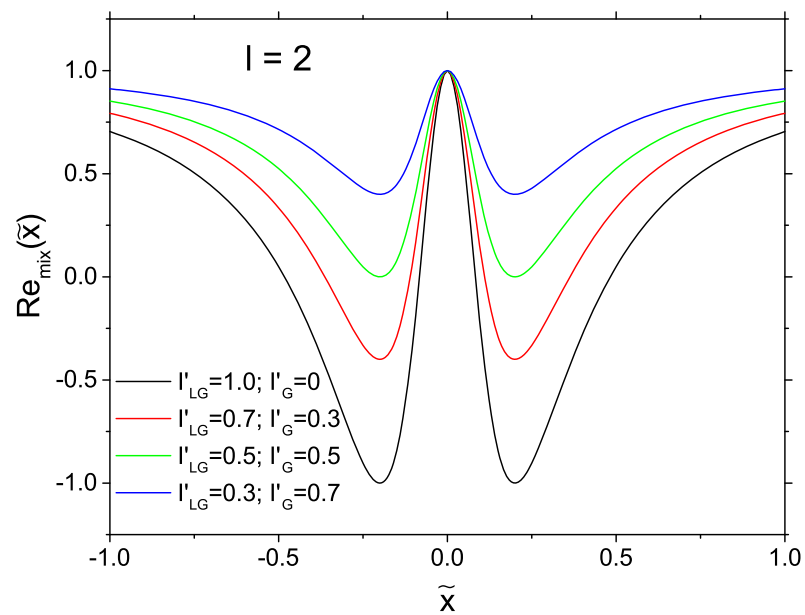

FIG. 5. Weight average $\mathrm{Re}_{\text {mix }}$ of the real part of the complex degrees of coherence of a pure Gaussian beam mixed with a $l=1$ (left) and a $l=2$ (right) Laguerre-Gauss beam, as a function of the normalized coordinate $\tilde{x} . I_{G}^{\prime}=I_{G} /\left(I_{G}+I_{\mathrm{LG}}\right)$ and $I_{\mathrm{LG}}^{\prime}=I_{\mathrm{LG}} /\left(I_{G}+I_{\mathrm{LG}}\right)$ are normalized intensities. $\operatorname{Re}_{\text {mix }}$ has been computed for $\tilde{y_{0}}=0.2$. 


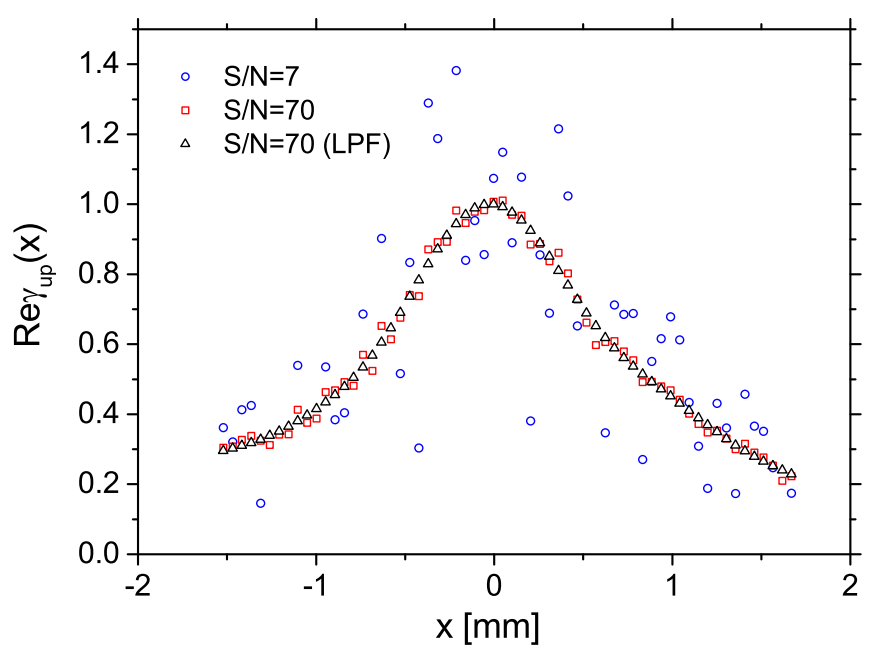

FIG. 6. Effect of random noise on the topological profile $\Delta X=$ $1.56 \mathrm{~mm}$ of Fig. 4 for different $S / N$. The curve $S / N=70$ (LPF) has been obtained using a low-pass filter with cutoff frequency $\nu=1.5 \mathrm{~mm}^{-1}$ applied to the curve $S / N=70$ (red squares).

intensities. Notice that the minima of $\mathrm{Re}_{\text {mix }}$ are given by $\left(I_{G}-I_{\mathrm{LG}}\right) /\left(I_{G}+I_{\mathrm{LG}}\right)$ and the maximum range is for $I_{G}=0$.

We investigate how random noise affects the topological profiles by using the proposed analysis. A random noise $N(x)$ has been added to the pattern obtained with the double-slit with spacing $\Delta X=1.56 \mathrm{~mm}$. The noise level has been changed in order to tune the signal-to-noise ratio, defined as $S / N=P_{\max } /\left(\sqrt{2} \sigma_{N}\right)$, where $P_{\max }$ is the maximum intensity of $P(x)$ in the observation region and $\sigma_{N}$ is the root-mean-square of $N(x)$. As in the previous case Eq. (3) and Eq. (2) have been used to find the topological profiles. The results of the analysis for $l=1$ are shown in Fig. 6. Random noise poses some limitations about the reconstruction of the topological profiles when $\sqrt{2} \sigma_{N}$ is comparable to the beam intensity ( $S / N \leq 7$ in our case). By contrast, a good reconstruction is possible for high values of the signal-to-noise ratio as shown for $S / N=70$.

In our analysis we considered a symmetric condition obtained experimentally by placing the center of the double-slit on the singularity. However, the pointing instability can produce some important effects on the topological profiles. We investigate how the asymmetric configurations change the analysis by introducing a displacement parameter $b$, defined as the vertical distance between the center of the double-slit and the singularity. The $b$ parameter is used to define a corresponding parametric function $\epsilon(x ; b)=\operatorname{Re} \gamma_{\text {slit }}^{A}(x ; b)-\operatorname{Re} \gamma_{\text {slit }}$, which provides the absolute discrepancy between the asymmetric and the symmetric configurations. $\operatorname{Re} \gamma_{\text {slit }}^{A}(x ; b)$ is the real part of the complex degree of coherence measured along the double-slit center in the asymmetric configuration $b \neq 0$. By computing the parametric function $\epsilon(x ; b)$ we can always determine (for a given $b$ ) the function $\operatorname{Re} \gamma_{\text {slit }}^{A}(x ; b)$ from $\operatorname{Re} \gamma_{\text {slit }}$ and vice versa.

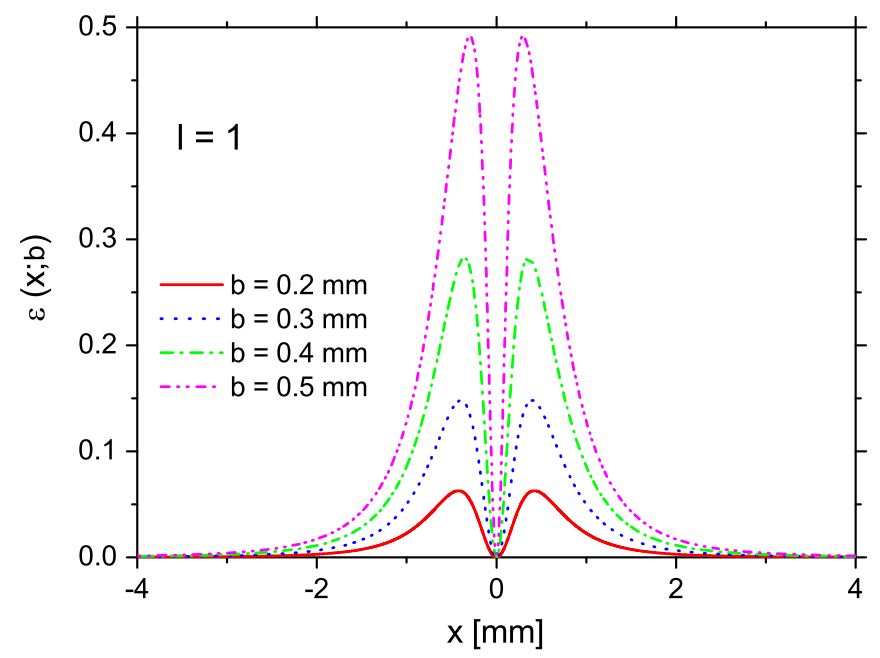

FIG. 7. Parametric function $\epsilon(x ; b)$ for a double-slit with spacing $\Delta X=1.25 \mathrm{~mm}$.

The parametric function shown in Fig. 7 has been computed for $l=1$ by using Eq. (A1), and the corresponding functions $\operatorname{Re}_{\text {slit }}^{A}(x ; b)$ are shown in Fig. 8. Results show that the number of maxima and minima are conserved by changing $b$, thus the topological charge is still identifiable also with a large displacement $b=0.5 \mathrm{~mm}$ (the slit separation used in calculation was $\Delta X=1.25 \mathrm{~mm}$ ). On the contrary, the shape of $\operatorname{Re} \gamma_{\text {slit }}^{A}(x ; b)$ is appreciably different with respect to the symmetric case $(b=0)$. The difference depends on the value of the parameter $b$ and should be taken into account for precise phase reconstruction.

This method exploits simple apertures thus can be advantageously extended to lower wavelengths in the $\mathrm{x}$-ray range. The main limitation in the hard $\mathrm{x}$-ray region is the distance necessary to overlap the diffracted field from the slits. For example at $12 \mathrm{keV}$ with a slit separation

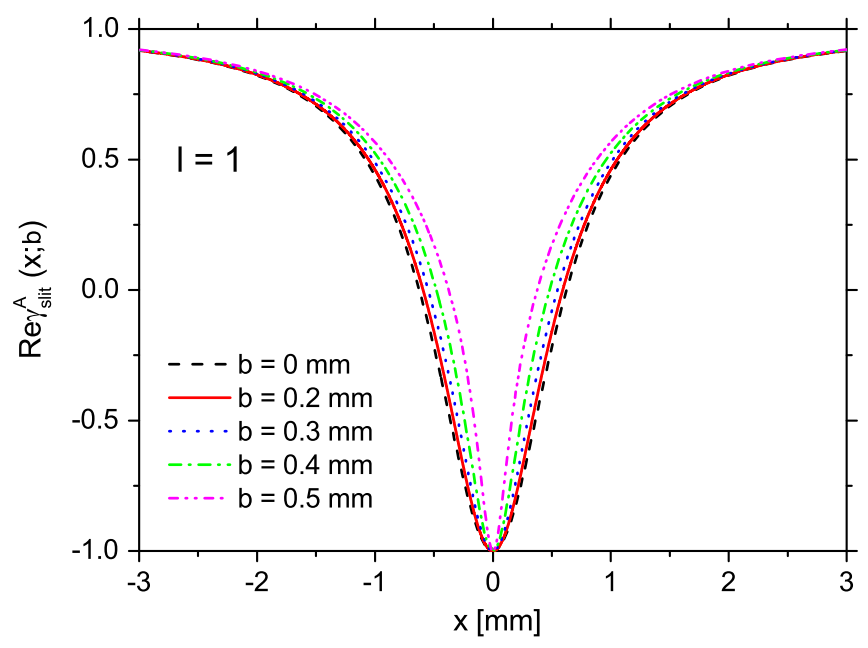

FIG. 8. Real part of the complex degree of coherence $\operatorname{Re} \gamma_{\text {slit }}^{A}(x ; b)$ measured along the double-slit center in asymmetric configuration. The black dashed line is $\operatorname{Re} \gamma_{\text {slit }}(x)$. 
of $100 \mu \mathrm{m}$ the far-field observation distance should be much larger than $100 \mathrm{~m}$, which represents a real limit in many $x$-ray facilities. Fortunately, this problem can be overcome by using $\mathrm{x}$-ray optical elements as compound lenses or x-rays mirrors $[19,20]$ in order to decrease the observation distance from the interferometer. A far field observation distance less than $5 \mathrm{~m}$ has been proven to be appropriate to obtain high-quality interference pattern in a X-ray Young experiment up to $25 \mathrm{keV}$ [21]. Thus the ALC scheme, coupled to available compound refractive lenses is expected to yield reliable topological profiles up to $25 \mathrm{keV}$ photons $(\lambda \sim 0.5 \AA)$.

\section{CONCLUSIONS}

We have shown that the topological profiles of the two dimensional maps of the asymmetric lateral coherence, which so far have been obtained by a complete scan of a double-slit array, can be measured in single-shot by exploiting a single double-slit centered on the vortex singularity. The phase properties of the vortex beam are in good agreement with theory close to the singularity. Despite far from the singularity experimental limitations prevent a quantitative comparison, the topological charge of the vortex can be easily recognized from the different oscillatory behavior of the profiles, as probed for the cases of $l=1$ and $l=2$. Moreover, we find that for precise wavefront reconstruction the acquired patterns should have a good level of signal-to-noise ratio $(S / N \gg 10)$, while the mixing of the vortex beam with a pure Gaussian beam yields a loss of visibility of the minima of $\mathrm{Re}_{\text {mix }}$, which reduces the sensibility to recognize the vortex, depending on the relative intensities of the beams. On the contrary, the pointing instability does not reduce this sensibility, despite the topological profiles should be correct with the parametric function $\epsilon(x ; b)$ for precise phase reconstruction.

\section{APPENDIX: DERIVATION OF EQ. (2)}

Let us consider the complex degree of coherence

$$
\gamma_{c}\left(\vec{x}_{j}, \vec{x}_{k}\right)=\left|\gamma_{c}\left(\vec{x}_{j}, \vec{x}_{k}\right)\right| e^{i \phi_{j k}}
$$

where $\vec{x}_{j}, \vec{x}_{k}$ are two arbitrary points on the detection plane sketched in Fig. 9 and $\phi_{j k}=\phi_{j}-\phi_{k}$ the phase difference between the complex fields in $\vec{x}_{j}, \vec{x}_{k}$.

By considering a fully coherent radiation $\left|\gamma_{c}\left(\vec{x}_{j}, \vec{x}_{k}\right)\right|=1$ we write [11]

$$
e^{i \phi_{a b}} e^{i \phi_{b c}} e^{i \phi_{c d}} e^{i \phi_{d a}}=1
$$

where the indices $a, b, c, d$ are related to the points $A=\left(0,-y_{0}\right), B=\left(0, y_{0}\right), C=\left(x, y_{0}\right), D=\left(x,-y_{0}\right)$ as shown in Fig. 9.

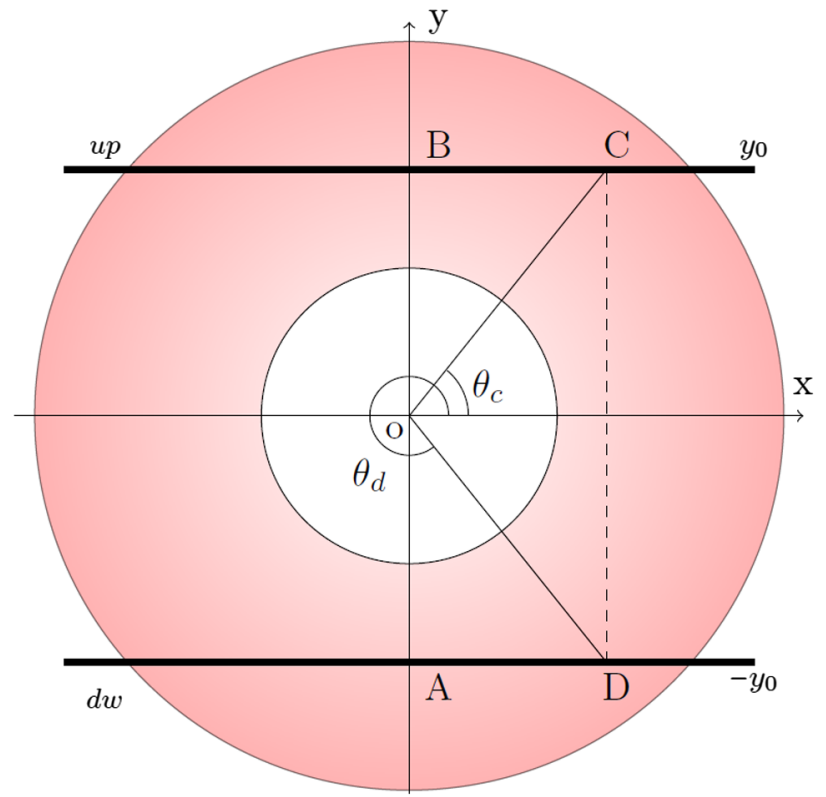

FIG. 9. Geometry of the single-shot technique. An horizontal double-slit (up and dw) with spacing $2 y_{0}$ is centered on the vortex singularity at the detection plane. The points $C=\left(x, y_{0}\right)$ and $D=\left(x,-y_{0}\right)$ are identified by the angles $\theta_{c}$ and $\theta_{d}$, respectively. The points $A=\left(0,-y_{0}\right)$ and $B=\left(0, y_{0}\right)$ are fixed and are identified by the angles $\theta_{a}=-\pi / 2$ and $\theta_{b}=\pi / 2$ not shown in the figure.

Explicitly we get

$$
e^{i \phi_{a b}}=\exp \left\{i l\left(\theta_{a}-\theta_{b}\right)\right\}=\exp \{i l \pi\},
$$

$$
\begin{aligned}
e^{i \phi_{b c}}= & \exp \left\{i l\left(\theta_{b}-\theta_{c}\right)\right\}=\exp \left\{-i l \cdot \arccos \left(\frac{x}{\sqrt{x^{2}+y_{0}^{2}}}\right)\right\} \\
& \times \exp \left\{i l \frac{\pi}{2}\right\},
\end{aligned}
$$

$$
e^{i \phi_{c d}}=\exp \left\{i l\left(\theta_{c}-\theta_{d}\right)\right\}=\exp \left\{2 i l \cdot \arccos \left(\frac{x}{\sqrt{x^{2}+y_{0}^{2}}}\right)\right\}
$$

$$
\begin{aligned}
e^{i \phi_{d a}}= & \exp \left\{i l\left(\theta_{d}-\theta_{a}\right)\right\}=\exp \left\{-i l \cdot \arccos \left(\frac{x}{\sqrt{x^{2}+y_{0}^{2}}}\right)\right\} \\
& \times \exp \left\{-i l \frac{\pi}{2}\right\} .
\end{aligned}
$$

Substituting Eq. (A3)-(A6) into Eq. (A2) we obtain

$$
\begin{aligned}
& \exp \left\{2 i l \cdot \arccos \left(\frac{x}{\sqrt{x^{2}+y_{0}^{2}}}\right)\right\} \\
& =\exp \left\{2 i l \cdot \arccos \left(\frac{x}{\sqrt{x^{2}+y_{0}^{2}}}\right)\right\} \exp \{-i l \pi\} .
\end{aligned}
$$


Independently, from Eq. (A1) we find

$$
\gamma_{\text {slit }}(x)=\exp \left\{2 i l \cdot \arccos \left(\frac{x}{\sqrt{x^{2}+y_{0}^{2}}}\right)\right\}
$$

and

$$
\gamma_{u p}\left(x, x_{0}=0\right)=\exp \left\{i l \cdot \arccos \left(\frac{x}{\sqrt{x^{2}+y_{0}^{2}}}\right)\right\},
$$

thus from Eq. (A7)-(A9) we obtain

$$
\gamma_{\text {slit }}(x)=\left[\gamma_{u p}(x)\right]^{2} e^{-i l \pi} .
$$

Similarly, by considering the complex degree of coherence $\gamma_{d w}(x)$ along the slit below the singularity we obtain

$$
\gamma_{\text {slit }}(x)=\left[\gamma_{d w}(x)^{*}\right]^{2} e^{-i l \pi},
$$

where $*$ denotes the complex conjugate.

The real part of Eq. (A10) and Eq. (A11) gives

$$
\begin{aligned}
\operatorname{Re} \gamma_{\text {slit }} & =\left[2\left(\operatorname{Re} \gamma_{u p}\right)^{2}-1\right] \cos (l \pi) \\
& =\left[2\left(\operatorname{Re} \gamma_{d w}^{*}\right)^{2}-1\right] \cos (l \pi),
\end{aligned}
$$

and hence

$$
\begin{aligned}
\left|\operatorname{Re} \gamma_{u p}\left(x, x_{0}=0\right)\right| & =\left|\operatorname{Re} \gamma_{d w}^{*}\left(x, x_{0}=0\right)\right| \\
& =\frac{1}{\sqrt{2}} \sqrt{1+\frac{\operatorname{Re} \gamma_{\text {slit }}(x)}{\cos (l \pi)}} .
\end{aligned}
$$

[1] L. Allen, M. W. Beijersbergen, R. J. C. Spreeuw, and J. P. Woerdman, Orbital angular momentum of light and the transformation of Laguerre-Gaussian laser modes, Phys. Rev. A 45, 8185 (1992).

[2] Y. Yan, G. Xie, M. P. J. Lavery, H. Huang, N. Ahmed, C. Bao, Y. Cao, L. Li, Z. Zhao, A. F. Molisch, M. Tur, M.J. Padgett, and A. E. Willner, High-capacity millimetre-wave communications with orbital angular momentum multiplexing, Nat. Commun. 5, 4876 (2014).

[3] F. Tamburini, G. Anzolin, G. Umbriaco, A. Bianchini, and C. Barbieri, Overcoming the Rayleigh Criterion Limit with Optical Vortices, Phys. Rev. Lett. 97, 163903 (2006).

[4] Y. Ma, G. Rui, B. Gu, and Y. Cui, Trapping and manipulation of nanoparticles using multifocal optical vortex metalens, Sci. Rep. 7, 14611 (2017).

[5] D. Mawet, E. Serabyn, K. Liewer, R. Burruss, J. Hickey, and D. Shemo, The vector vortex coronagraph: laboratory results and first light at Palomar observatory, Astrophys. J. 709, 53 (2010).

[6] A. Picón, J. Mompart, J. R. de Aldana, L. Plaja, G. F. Calvo, and L. Roso, Photoionization with orbital angular momentum beams, Opt. Express 18, 3660 (2010).
[7] V. Petrillo, G. Dattoli, I. Drebot, and F. Nguyen, Compton Scattered X-Gamma Rays with Orbital Momentum, Phys. Rev. Lett. 117, 123903 (2016).

[8] M. Katoh, M. Fujimoto, N. S. Mirian, T. Konomi, Y. Taira, T. Kaneyasu, M. Hosaka, N. Yamamoto, A. Mochihashi, Y. Takashima, K. Kuroda, A. Miyamoto, K. Miyamoto, and S. Sasaki, Helical phase structure of radiation from an electron in circular motion, Sci. Rep. 7, 6130 (2017).

[9] S. V. Abdrashitov, O. V. Bogdanov, P. O. Kazinski, and T. A. Tukhfatullin, Orbital angular momentum of channeling radiation from relativistic electrons in thin $\mathrm{Si}$ crystal, Phys. Lett. A 382, 3141 (2018).

[10] V. Epp, J. Janz, and M. Zotova, Angular momentum of radiation at axial channeling, Nucl. Instrum. Methods Phys. Res., Sect. B 436, 78 (2018).

[11] B. Paroli, A. Cirella, I. Drebot, V. Petrillo, M. Siano, and M. A. C. Potenza, Asymmetric lateral coherence of OAM radiation reveals topological charge and local curvature, J. Opt. 20, 075605 (2018).

[12] B. Paroli, E. Chiadroni, M. Ferrario, A. Mostacci, V. Petrillo, M. A. C. Potenza, A. R. Rossi, and L. Serafini, Coherence properties and diagnostics of betatron radiation emitted by an externally-injected electron beam propagating in a plasma channel, Nucl. Instrum. Methods Phys. Res., Sect. B 355, 217 (2015).

[13] B. Paroli, E. Chiadroni, M. Ferrario, V. Petrillo, M. A. C. Potenza, A. R. Rossi, L. Serafini, and V. Shpakov, Asymmetric lateral coherence of betatron radiation emitted in laserdriven light sources, Europhys. Lett. 111, 44003 (2015).

[14] B. Paroli, E. Chiadroni, M. Ferrario, and M. A. C. Potenza, Analogical optical modeling of the asymmetric lateral coherence of betatron radiation, Opt. Express 23, 29912 (2015).

[15] B. Paroli, E. Bravin, S. Mazzoni, G. Trad, and M. A. C. Potenza, A modified two-slit interferometer for characterizing the asymmetric lateral coherence of undulator radiation, Europhys. Lett. 115, 14004 (2016).

[16] B. Paroli, E. Chiadroni, M. Ferrario, and M. A. C. Potenza, A systematic study of the asymmetric lateral coherence of radiation emitted by ultra-relativistic particles in laserdriven accelerators, Nucl. Instrum. Methods Phys. Res., Sect. A 839, 1 (2016).

[17] B. Paroli and M. A. C. Potenza, Two dimensional mapping of the asymmetric lateral coherence of thermal light, Opt. Express 24, 25676 (2016).

[18] B. Paroli, M. Siano, and M. A.C. Potenza, Asymmetric lateral coherence allows precise wavefront characterization, Europhys. Lett. 122, 44001 (2018).

[19] G. Cappuccio, S. B. Dabagov, D. Hampai, Y. I. Dudchik, and F. F. Komarov, A spherical compound refractive lens to control x-ray beams, International Conference on Charged and Neutral Particles Channeling Phenomena II, Proc. SPIE Int. Soc. Opt. Eng. 6634, 66341M (2007).

[20] I. N. Bukreeva, S. B. Dabagov, and S. Lagomarsino, Efficiency of an elliptically shaped x-ray mirror, Appl. Opt. 43, 6270 (2004).

[21] M. Lyubomirskiy, I. Snigireva, and A. Snigirev, Lens coupled tunable Young's double pinhole system for hard $\mathrm{X}$-ray spatial coherence characterization, Opt. Express 24, 13679 (2016). 